\title{
ON THE FUNCTIONAL LIMITS FOR PARTIAL SUMS UNDER STABLE LAW
}

\author{
KHURELBAATAR GONCHIGDANZAN AND KAMIL MARCIN KOSIŃSKI
}

\begin{abstract}
For the partial sums $\left(S_{n}\right)$ of independent random variables we define a stochastic process $s_{n}(t):=\left(1 / d_{n}\right) \sum_{k \leq[n t]}\left(S_{k} / k-\mu\right)$ and prove that

$$
(1 / \log N) \sum_{n \leq N}(1 / n) \mathbf{I}\left\{s_{n}(t) \leq x\right\} \rightarrow G_{t}(x) \text { a.s. }
$$

if and only if $(1 / \log N) \sum_{n \leq N}(1 / n) \mathbb{P}\left(s_{n}(t) \leq x\right) \rightarrow G_{t}(x)$, for some sequence $\left(d_{n}\right)$ and distribution $G_{t}$. We also prove an almost sure functional limit theorem for the product of partial sums of i.i.d. positive random variables attracted to an $\alpha$-stable law with $\alpha \in(1,2]$.
\end{abstract}

\section{INTRODUCTION AND MAIN RESULT}

In the past two decades many interesting extensions of the classical central limit theorem (CLT) have been obtained. One of the extensions is known as almost sure central limit theorem (ASCLT) which is discovered by Brosamler (1988) and Schatte (1988) and has been extensively studied for independent random variables as well as dependent variables. Motivated by ASCLT, almost sure versions of many limit theorems in probability and statistics have been obtained in the past. It is known that for i.i.d. r.v.'s ASCLT holds under the same assumptions as CLT but in general, the existence of the weak limit does not always imply the almost sure limiting result. For more discussions about the early results on ASCLT we refer to Berkes [1].

In this note we consider the product of partial sums, denoted by $S_{n}$, of a sequence of random variables attracted to a stable distribution and its limit distributions. Rempała and Wesolowski [9] established the limit distribution of the product of partial sums of a sequence of i.i.d. positive r.v.'s with mean $\mu$ and variance $\sigma^{2}$ :

$$
\left(\frac{\prod_{k=1}^{n} S_{k}}{n ! \mu^{n}}\right)^{\mu /(\sigma \sqrt{n})} \stackrel{d}{\rightarrow} e^{\sqrt{2} \mathcal{N}(0,1)}
$$

Zhang and Huang [11] proves a weak invariance principle of (1) for i.i.d. r.v.'s. Recently, Kosiński [8] has shown that the weak invariance principle still holds when the partial sums are attracted to an $\alpha$-stable law with $\alpha \in(1,2]$ which also generalizes the earlier result by Qi [10].

Date: May 7, 2009.

2000 Mathematics Subject Classification. Primary G0F05.

Key words and phrases. Almost sure limit theorem, Logarithmic average, Stable law, Product of partial sums. 
Throughout this paper, $\log \log x$ and $\log x$ stand for $\ln \ln \left(\max \left\{x, e^{e}\right\}\right)$ and $\ln (\max \{x, e\})$ respectively. We also use the notations $a_{n} \ll b_{n}$ for $a_{n}=O\left(b_{n}\right)$ and $\mathbf{I}(A)$ for the indicator function on a set $A$.

Our main result in this note is to establish an almost sure version of the result by Kosiński [8] that can generalize the early results by Gonchigdanzan and Rempała [4] and Gonchigdanzan [5, 6].

Recall that a sequence of and i.i.d. r.v.'s $\left\{X_{n}: n \geq 1\right\}$ is said to be in the domain of attraction of a stable law $\mathcal{L}$ if there exist sequences $\left(a_{n}\right)$ and $\left(b_{n}\right)$ such that

$$
\frac{S_{n}-b_{n}}{a_{n}} \stackrel{d}{\rightarrow} \mathcal{L}_{\alpha}
$$

where $\mathcal{L}_{\alpha}$ is one of the stable distributions with index $\alpha \in(0,2]$. Moreover, let $\left\{\mathcal{L}_{\alpha}(s)\right.$ : $s \geq 0\}$ be the $\alpha$-stable Lévy process corresponding to $\mathcal{L}_{\alpha}$, that is $\mathcal{L}_{\alpha}(1) \stackrel{d}{=} \mathcal{L}_{\alpha}$.

The following theorem is well known (see, e.g., Hall [7]).

Theorem 1 (Stability Theorem). The general stable law is given, to within type, by a characteristic function of one of the following forms:

(1) $\phi(t)=\exp \left(-t^{2} / 2\right)$ (normal case, $\left.\alpha=2\right)$;

(2) $\phi(t)=\exp \left(-|t|^{\alpha}\left(1-i \beta \operatorname{sgn}(t) \tan \left(\frac{1}{2} \pi \alpha\right)\right)\right)(0<\alpha<1$ or $1<\alpha<2,-1 \leq \beta \leq 1)$;

(3) $\phi(t)=\exp (-|t|(1+i \beta \operatorname{sgn}(t) 2 / \pi \log |t|)(\alpha=1,-1 \leq \beta \leq 1)$.

It is worth mentioning that in Theorem 1, $\beta$ is the skewness parameter. In our paper, $\beta=1$ since $X_{1}$ is a positive random variable.

The first result of this note is the following almost sure functional limit theorem:

Theorem 2. Let $\left\{X_{n}: n \geq 1\right\}$ be a sequence of i.i.d. positive random variables with $\mathbb{E} X_{1}=\mu$ in the domain of attraction of an $\alpha$-stable law $\mathcal{L}_{\alpha}$ with $\alpha \in(1,2]$ and characteristic function as in Theorem 1. Define a process $\left\{\pi_{n}(t): 0 \leq t \leq 1\right\}$ by

$$
\pi_{n}(t):=\left(\prod_{k=1}^{[n t]} \frac{S_{k}}{\mu k}\right)^{\mu / a_{n}}
$$

where $\left(a_{n}\right)$ is a sequence of positive numbers that satisfies $\left(S_{n}-\mu n\right) / a_{n} \stackrel{d}{\rightarrow} \mathcal{L}_{\alpha}$ as $n \rightarrow \infty$. Then for any real $x$

$$
\frac{1}{\log N} \sum_{n=1}^{N} \frac{1}{n} \mathbf{I}\left(\pi_{n}(t) \leq x\right) \stackrel{a . s .}{\rightarrow} F_{t}(x) \text { as } N \rightarrow \infty
$$

where $F_{t}$ is the distribution function of the random variable $\exp \left(\int_{0}^{t} \frac{\mathcal{L}_{\alpha}(s)}{s} d s\right)$.

Remark 1. If $X_{1}$ has finite variance equal to $\sigma^{2}$ then $\alpha=2, \mathcal{L}_{\alpha} \stackrel{d}{=} \mathcal{N}(0,1)$ and $a_{n} \sim \sigma \sqrt{n}$, thus Theorem 2 implies the main result of Gonchigdanzan [6] which in particular yields the result of Gonchigdanzan and Rempała [4] Theorem 2 since it is easy to verify that

$$
\int_{0}^{1} \frac{\mathcal{L}_{\alpha}(s)}{s} d s \stackrel{d}{=} \sqrt{2} \mathcal{N}(0,1)
$$


Moreover, Kosiński [8] showed that for any $\alpha \in(1,2]$

$$
\int_{0}^{1} \frac{\mathcal{L}_{\alpha}(s)}{s} d s \stackrel{d}{=}(\Gamma(\alpha+1))^{1 / \alpha} \mathcal{L}_{\alpha}
$$

hence Theorem 2 also yields the result of Gonchigdanzan [5] Theorem 1.1.

Our next result is the following Berkes-Dehling type of theorem (Berkes and Dehling 2, Theorem 2]).

Theorem 3. Let $\left\{Y_{n}: n \geq 1\right\}$ be a sequence of independent random variables and $S_{n}=$ $Y_{1}+\cdots+Y_{n}$. Let $\left(d_{n}\right)$ be a sequence of positive numbers such that

(2) $\quad \frac{d_{l}}{d_{k}} \gg\left(\frac{l}{k}\right)^{\gamma} \quad\left(l \geq k \geq n_{0}\right)$

for some $\gamma>0$ and $n_{0} \geq 1$ and

$$
\mathbb{E}\left|\frac{S_{n}-\mu n}{d_{n}}\right| \ll e^{\gamma^{\prime}(\log n)^{1-\varepsilon}}
$$

for some constant $\mu$ and $\gamma^{\prime} \in(0, \gamma)$. Then for any distribution $G_{t}$,

$$
\frac{1}{\log N} \sum_{n=1}^{N} \frac{1}{n} \mathbf{I}\left(\frac{1}{d_{n}} \sum_{k=1}^{[n t]}\left(\frac{S_{k}}{k}-\mu\right) \leq x\right) \stackrel{\text { a.s. }}{\rightarrow} G_{t}(x) \text { as } \quad N \rightarrow \infty
$$

if and only if

$$
\frac{1}{\log N} \sum_{n=1}^{N} \frac{1}{n} \mathbb{P}\left(\frac{1}{d_{n}} \sum_{k=1}^{[n t]}\left(\frac{S_{k}}{k}-\mu\right) \leq x\right) \rightarrow G_{t}(x) \text { as } N \rightarrow \infty .
$$

\section{Auxiliary Results}

The following three lemmas are needed for the proof of our main result.

Lemma 4 (Lemma 2.3, Gonchigdanzan [5]). Under the assumption of Theorem 2 we have

$$
\left|\frac{\mu}{a_{n}} \sum_{k=1}^{[n t]} \log \left(\frac{S_{k}}{\mu k}\right)-\frac{1}{a_{n}} \sum_{k=1}^{[n t]}\left(\frac{S_{k}}{k}-\mu\right)\right| \stackrel{\text { a.s. }}{\rightarrow} 0 \quad \text { as } \quad n \rightarrow \infty .
$$

Lemma 5. Under the assumptions of Theorem 2 we have

$$
\frac{1}{a_{n}} \sum_{k=1}^{[n t]}\left(\frac{S_{k}}{k}-\mu\right) \stackrel{d}{\rightarrow} \int_{0}^{t} \frac{\mathcal{L}_{\alpha}(s)}{s} d s \quad \text { in } \quad D[0,1] .
$$

Proof. This is a particular case of Theorem 2 in Kosiński [8] when $f(x)=x$.

Lemma 6. Under the assumptions of Theorem 3 we have

$$
\mathbb{E}\left(\frac{1}{d_{n}} \max _{1 \leq k \leq n}\left|\sum_{j=1}^{k} \log \left(\frac{n+1}{j}\right)\left(Y_{j}-\mu\right)\right|\right) \ll \log n \mathbb{E}\left(\frac{1}{d_{n}} \max _{1 \leq k \leq n}\left|S_{k}-k \mu\right|\right) \ll \log n e^{\gamma^{\prime}(\log n)^{1-\varepsilon}} .
$$

Proof. The first part is Lemma 1 in Gonchigdanzan [6] valid for any sequence of random variables. The second part is Lemma 1 in Berkes and Dehling [2] combined with the assumption (3). 


\section{Proofs of the MAIN Results}

To prove Theorem 2 we need the result in Theorem 3 , Let us prove Theorem 3 first, then Theorem 2 for convenience.

Proof of Theorem [3. According to Berkes and Dehling [2] (p. 1647) it suffices to prove that for any bounded Lipschitz function $g$ on $D[0,1]$ we have

$$
\frac{1}{\log n} \sum_{k=1}^{n} \frac{1}{k}\left(g\left(\frac{s_{k}}{d_{k}}\right)-\mathbb{E} g\left(\frac{s_{k}}{d_{k}}\right)\right) \stackrel{\text { a.s. }}{\rightarrow} 0 \text { as } n \rightarrow \infty,
$$

where $s_{n}:=s_{n}(t)=\sum_{k \leq[n t]}\left(S_{k} / k-\mu\right)$.

It turns out that the following estimate is indeed sufficient for (6) (see p.1648 [2] for the proof):

$$
\mathbb{E}\left(\sum_{k=1}^{n} \frac{1}{k} \xi_{k}\right)^{2} \ll \log ^{2} n(\log \log n)^{-1-\varepsilon} \text { for some } \varepsilon>0,
$$

where $\xi_{k}=g\left(s_{k} / d_{k}\right)-\mathbb{E} g\left(s_{k} / d_{k}\right)$.

Observe that $\sum_{k=1}^{n}\left(S_{k} / k-\mu\right)=\sum_{k=1}^{n} b_{k, n}\left(Y_{k}-\mu\right)$ where $b_{k, n}=\sum_{j=k}^{n} 1 / j$. It can be easily seen that

$$
s_{l}-s_{k}=b_{[k t]+1,[l t]}\left(S_{[k t]}-[k t] \mu\right)+\left(b_{[k t]+1,[l t]}\left(Y_{[k t]+1}-\mu\right)+\cdots+b_{[l t],[l t]}\left(Y_{[l t]}-\mu\right)\right)
$$

for $l \geq k$.

Obviously $s_{l}-s_{k}-b_{[k t]+1,[t t]}\left(S_{[k t]}-\mu[k t]\right)$ is independent of $s_{k}$, so we get

$$
\operatorname{Cov}\left(g\left(\frac{s_{k}}{d_{k}}\right), g\left(\frac{s_{l}-s_{k}-b_{[k t]+1,[l t]}\left(S_{[k t]}-\mu[k t]\right)}{d_{l}}\right)\right)=0 \text { for } l \geq k .
$$

Since $g$ is a bounded Lipschitz it follows that

$$
\begin{aligned}
\left|\mathbb{E}\left(\xi_{k} \xi_{l}\right)\right| & =\mid \operatorname{Cov}\left(g\left(\frac{s_{k}}{d_{k}}\right), g\left(\frac{s_{l}}{d_{l}}\right)-g\left(\frac{s_{l}-s_{k}-b_{[k t]+1,[l t]}\left(S_{[k t]}-\mu[k t]\right)}{d_{l}}\right) \mid\right. \\
& \ll \mathbb{E}\left(\max _{0 \leq t \leq 1} \frac{\left|s_{k}+b_{[k t]+1,[l t]}\left(S_{[k t]}-\mu[k t]\right)\right|}{d_{l}}\right) \\
& \leq \mathbb{E}\left(\max _{0 \leq t \leq 1} \frac{\left|s_{k}\right|}{d_{l}}\right)+\mathbb{E}\left(\max _{0 \leq t \leq 1} \frac{\left|b_{[k t]+1,[l t]}\left(S_{[k t]}-\mu[k t]\right)\right|}{d_{l}}\right) \\
& =\frac{d_{k}}{d_{l}}\left(\mathbb{E}\left(\max _{0 \leq t \leq 1} \frac{\left|s_{k}\right|}{d_{k}}\right)+\mathbb{E}\left(\max _{0 \leq t \leq 1} b_{[k t]+1,[l t]} \frac{\left|S_{[k t]}-\mu[k t]\right|}{d_{k}}\right)\right) .
\end{aligned}
$$

Moreover, noticing $\max _{0 \leq t \leq 1} b_{[k t]+1,[l t]}=\log (l / k)$ and applying Lemma 6 we get

$$
\begin{aligned}
\left|\mathbb{E}\left(\xi_{k} \xi_{l}\right)\right| & \ll \frac{d_{k}}{d_{l}}\left(\mathbb{E}\left(\max _{0 \leq t \leq 1} \frac{1}{d_{k}}\left|\sum_{i=1}^{[k t]} b_{i, k}\left(Y_{i}-\mu\right)\right|\right)+\log (l / k) \mathbb{E}\left(\max _{0 \leq t \leq 1} \frac{\left|S_{[k t]}-\mu[k t]\right|}{d_{k}}\right)\right) \\
& =\frac{d_{k}}{d_{l}}\left(\mathbb{E}\left(\max _{0 \leq j \leq k} \frac{1}{d_{k}}\left|\sum_{i=1}^{j} b_{i, k}\left(Y_{i}-\mu\right)\right|\right)+\log (l / k) \mathbb{E}\left(\max _{0 \leq j \leq k} \frac{\left|S_{j}-\mu j\right|}{d_{k}}\right)\right) \\
& \ll \log l \frac{d_{k}}{d_{l}} \mathbb{E}\left(\max _{1 \leq j \leq k} \frac{\left|S_{j}-\mu j\right|}{d_{k}}\right) \ll \log l\left(\frac{k}{l}\right)^{\gamma} \log k e^{\gamma^{\prime}(\log k)^{1-\varepsilon}}=: c_{k, l} .
\end{aligned}
$$


On the other hand we also have $\mathbb{E}\left(\xi_{k} \xi_{l}\right) \ll 1$ because $\xi_{k}$ is bounded. Hence we estimate $\mathbb{E}\left(\xi_{k} \xi_{l}\right)$ as follows:

$$
\mathbb{E}\left(\xi_{k} \xi_{l}\right) \ll\left\{\begin{aligned}
1, & \text { if } l / k \leq \exp \left((\log n)^{1-\varepsilon}\right) \\
c_{k, l}, & \text { if } l / k \geq \exp \left((\log n)^{1-\varepsilon}\right)
\end{aligned}\right.
$$

where $\varepsilon$ is any positive number.

Thus we get

$$
\sum_{\substack{1 \leq k \leq l \leq n \\ l / k \leq \exp \left((\log n)^{1-\varepsilon}\right)}} \frac{\mathbb{E}\left(\xi_{k} \xi_{l}\right)}{k l} \leq \sum_{1 \leq k \leq n} \frac{1}{k} \sum_{k \leq l \leq k e^{(\log n)^{1-\varepsilon}}} \frac{1}{l} \ll \sum_{k=1}^{n} \frac{1}{k} \log ^{1-\varepsilon} n \ll \log ^{2-\varepsilon} n
$$

and

$$
\begin{aligned}
\sum_{\substack{1 \leq k \leq l \leq n \\
l / k \geq \exp \left((\log n)^{1-\varepsilon}\right)}} \frac{\mathbb{E}\left(\xi_{k} \xi_{l}\right)}{k l} & \leq \log ^{2} n e^{\gamma^{\prime}(\log n)^{1-\varepsilon}} \sum_{\substack{1 \leq k \leq l \leq n \\
l / k \geq \exp \left((\log n)^{1-\varepsilon}\right)}} \frac{1}{k l}\left(\frac{k}{l}\right)^{\gamma} \\
& \leq \log ^{2} n e^{\left(\gamma^{\prime}-\gamma\right)(\log n)^{1-\varepsilon}} \sum_{\substack{1 \leq k \leq l \leq n \\
k l}} \frac{1}{k l} \\
& \ll \log ^{4} n e^{\left(\gamma^{\prime}-\gamma\right)(\log n)^{1-\varepsilon}} \ll \log ^{2-\varepsilon} n,
\end{aligned}
$$

where the last estimation follows because $\gamma^{\prime} \in(0, \gamma)$.

Since

$$
\mathbb{E}\left(\sum_{k=1}^{n} \frac{1}{k} \xi_{k}\right)^{2} \ll \sum_{1 \leq k \leq l \leq n} \frac{1}{k l}\left|\mathbb{E}\left(\xi_{k} \xi_{l}\right)\right|
$$

by (8) and (9) it follows (7).

Before proving Theorem 2, recall that it is well known that the sequence $\left(a_{n}\right)$ in Theorem 2 can be written as $a_{n}=n^{1 / \alpha} L(n)$ where $L$ is a slowly varying function.

Proof of Theorem 2. We first show the equivalence of (4) and (51) under the conditions of Theorem 2 setting $d_{n}:=a_{n}$. In fact (3) is a direct consequence of Theorem 6.2 in DeAcosta and Giné [3]. (2) can be easily verified using the facts that $a_{n}=n^{1 / \alpha} L(n)$ and $L(k) / L(n) \ll(k / n)^{\varepsilon}$ for any $\varepsilon>0$ where $L$ is a slowly varying function. Thus by Theorem (3) (41) is equivalent to (5) with $Y_{n} \stackrel{d}{=} X_{n}$ satisfying the conditions of Theorem 2 , Now applying Lemma 4 and Lemma 5 we get

$$
\frac{1}{\log N} \sum_{n=1}^{N} \frac{1}{n} \mathbf{I}\left(\frac{\mu}{a_{n}} \sum_{k=1}^{[n t]} \log \left(\frac{S_{k}}{\mu k}\right) \leq x\right) \stackrel{\text { a.s. }}{\rightarrow} \mathbb{P}\left(\int_{0}^{t} \frac{\mathcal{L}_{\alpha}(s)}{s} d s \leq x\right) \quad \text { as } \quad N \rightarrow \infty .
$$

\section{REFERENCES}

[1] Berkes, I. (1998). Results and problems related to the pointwise central limit theorem. Asymptotic results in Probability and Statistics, (A volume in honour of Miklós Csörgö), 59-60, Elsevier, Amsterdam.

[2] Berkes, I., Dehling, H. (1993). Some limit theorems in log density. Annals of Probability $21,1640-1670$. 
[3] DeAcosta, A., Giné, E. (1979). Convergence of moments and related functionals in the general central limit theorem in Banach spaces. Z. Wahrsch. Verw. Gebiete. 48, 213-231.

[4] Gonchigdanzan, K., Rempała, G. (2006). A note on the almost sure limit theorem for the product of partial sums. Applied Mathematics Letters 19, 191-196.

[5] Gonchigdanzan, K. (2008). An almost sure limit theorem for the product of partial sums with stable distribution. Statistics and Probability Letters 78, 3170-3175.

[6] Gonchigdanzan, K. (2009). Almost sure functional limit theorem for the product of partial sums. ESAIM: Prob and Stat, doi:10.1051/ps:2008038.

[7] Hall P. (1981). A comedy of error: the canonical form for a stable characteristic function. Bull. London Math. Soc. 13, 23-28.

[8] Kosiński, K.M. (2009). On the functional limits of a function of partial sums. Stat. Probab. Lett., doi:10.1016/j.spl.2009.03.011.

[9] Rempała, G., Wesołowski, J. (2002). Asymptotics for products of sums and Ustatistics. Electronic Communication in Probability 7, 47-54.

[10] Qi, Y. (2003). Limit distributions for products of sums. Statistics \& Probability Letters $62,93-100$.

[11] Zhang, L. and Huang, W. (2007). A note on the invariance principle of the product of sums of random variables. Electronic Communication in Probability 12, 51-56.

Department of Mathematical Sciences, University of Wisconsin, Stevens Point, Wisconsin 54481, USA

E-mail address: hurlee@uwsp.edu

Korteweg-de Vries Institute for Mathematics, Universiteit van Amsterdam, Plantage MuiderGracht 24, 1018 TV Amsterdam, The Netherlands

Current address: Korteweg-de Vries Institute for Mathematics, University of Amsterdam, P.O. Box 94248, 1090 GE Amsterdam, The Netherlands and, EURANDOM, Technishe Universiteit Eindhoven, Postbus 513, 5600 MB Eindhoven, The Netherlands

E-mail address: K.M.Kosinski@uva.nl 